Artigo Original

Original Article

Fernanda Bastos de Andrade-Balieiro ${ }^{1}$

Renata Azevedo ${ }^{2}$

Brasília Maria Chiari²

Descritores

Fala

Sistema estomatognático

Voz

Tonsila palatina

Tonsila faríngea

Fonação

Keywords

Speech

Stomatognathic system

Voice

Palatine tonsil

Adenoids

Phonation

Endereço para correspondência: Fernanda Bastos de Andrade-Balieiro

R. Graúna, 180, Moema, São Paulo (SP),

Brasil, CEP: 04514-000.

E-mail: andrade.fe@gmail.com

Recebido em: 03/08/2011

Aceito em: 30/11/2011

\section{Aspectos do sistema estomatognático pré e pós-adenotonsilectomia}

\author{
Aspects of stomatognathic system before and \\ after adenotonsillectomy
}

\section{RESUMO}

Objetivo: Verificar os aspectos fonoaudiológicos do sistema estomatognático, incluindo as estruturas fonoarticulatórias (lábios, língua e palato mole) e as funções de deglutição, respiração, fala e fonação (qualidade vocal e ressonância), pré e pós adenotonsilectomia. Métodos: Participaram 22 crianças, 17 do gênero masculino e cinco do gênero feminino, na faixa etária entre 5 e 10 anos. Todas apresentavam hipertrofia de tonsilas palatina e faríngea com indicação cirúrgica de adenotonsilectomia e nenhuma delas havia passado por fonoterapia prévia. A avaliação fonoaudiológica foi realizada no período pré-operatório e entre um e seis meses após a adenotonsilectomia e constou de avaliação das estruturas (lábios, língua e palato mole), das funções de deglutição (líquido), respiração (modo), fala e fonação (qualidade vocal e ressonância). Para avaliar a qualidade vocal e a ressonância, participaram 15 sujeitos com avaliação pós-operatória realizada no período de um a dois meses. Resultados: Houve diferença quanto ao modo respiratório nasal, postura de lábios ocluídos em repouso, tônus de língua alterado, mobilidade adequada de palato mole, postura de língua alterada na deglutição de líquido, e ausência do mecanismo compensatório de interposição de lábios na deglutição. Houve redução na frequência dos processos de distorção. Em relação à fonação, foi observada discreta melhora na qualidade vocal e ressonância. Conclusão: Após a adenotonsilectomia algumas estruturas e funções podem se readaptar ou apresentar melhora espontaneamente. No entanto, foi necessário encaminhar a maioria das crianças para o atendimento fonoaudiológico na busca da readaptação das estruturas estomatognáticas e funções avaliadas.

\begin{abstract}
Purpose: To verify the speech therapy aspects of the stomatognathic system, including phonoarticulatory structures (lips, tongue, and soft palate) and swallowing, respiratory, speech, and phonation (vocal quality and resonance) functions, before and after undergoing adenotonsillectomy. Methods: The study included 22 children, 17 males and 5 females, aged between 5 and 10 years, suffering from hypertrophy of palatine and adenoid tonsils, with surgical indication for adenotonsillectomy and with no previous speech therapy. The speech-language pathology evaluation was performed before surgery and during the period between 1 and 6 months after adenotonsillectomy. It consisted of an evaluation of structures (lips, tongue, and soft palate) and of swallowing (liquid), respiration (mode), speech, and phonation (voice quality and resonance) functions. To evaluate vocal quality and resonance, 15 participants with the postoperative evaluation carried out in a period from 1 to 2 months were considered. Results: There were differences regarding nasal respiratory mode, lips closed at rest posture, changed tongue tonus, adequate mobility of the soft palate, changed tongue posture during liquid swallowing, and absence of interposition compensatory mechanism of lips in swallowing. Reduction in the frequency of distortion processes was also found. With regard to speech, little improvement in vocal quality and resonance was seen. Conclusion: Following adenotonsillectomy, some structures and functions can spontaneously readapt or improve. However, most children needed to be referred to speech therapy for readapting stomatognathic structures and the assessed functions.
\end{abstract}

Trabalho realizado no Programa de Pós-graduação em Distúrbios da Comunicação Humana: Campo Fonoaudiológico, Departamento de Fonoaudiologia, Universidade Federal de São Paulo - UNIFESP São Paulo (SP), Brasil.

(1) Programa de Pós-graduação em Distúrbios da Comunicação Humana: Campo Fonoaudiológico, Departamento de Fonoaudiologia, Universidade Federal de São Paulo - UNIFESP - São Paulo (SP), Brasil. (2) Departamento de Fonoaudiologia, Universidade Federal de São Paulo - UNIFESP - São Paulo (SP), Brasil. Conflito de interesse: nada a declarar. 


\section{INTRODUÇÃO}

A hipertrofia de tonsilas palatina e faríngea é a principal causa de obstrução mecânica da passagem de ar nas vias áreas superiores, ocasionando a respiração oral de suplência ${ }^{(1)}$. O padrão respiratório alterado pode proporcionar deformidades faciais, caracterizadas por alterações na maxila, palato duro, maloclusão, alteração na tonicidade e postura das estruturas do sistema estomatognático, no padrão de deglutição ${ }^{(2,3)} \mathrm{e}$ fala ${ }^{(4)}$. A mobilidade de palato mole, também, pode ficar prejudicada e alterar as características de ressonância vocal. Quando a qualidade de vida do sujeito fica prejudicada, muitas vezes a conduta médica volta-se para o procedimento cirúrgico.

A reavaliação, no período que compreende um mês até seis meses da data da cirurgia ${ }^{(2,5,6)}$, pode evidenciar melhora espontânea do padrão respiratório, vocal, de deglutição e readaptação das estruturas do sistema estomatognático, a qual é justificada pelo aumento de espaço na orofaringe associado a desobstrução das vias aéreas superiores, possibilitando a respiração nasal. Como consequência de um novo estímulo, uma redefinição dos articuladores e da ressonância vocal torna-se possível. No entanto, a persistência das alterações ${ }^{(3,7)}$ pode ocorrer em muitos casos pós adenotonsilectomia, e por isso a necessidade da intervenção fonoaudiológica para aquisição de um novo padrão.

O presente estudo teve como objetivo verificar os aspectos fonoaudiológicos do sistema estomatognático - incluindo estruturas fonoarticulatórias (lábios, língua e palato mole) e funções de deglutição, respiração, fala e fonação (qualidade vocal e ressonância), pré e pós-adenotonsilectomia.

\section{MÉTODOS}

O estudo foi aprovado pelo Comitê de Ética em Pesquisa da Universidade Federal de São Paulo (UNIFESP), sob o protocolo 1422/09. Todos os responsáveis pelos participantes assinaram o Termo de Consentimento Livre e Esclarecido. Participaram 22 crianças, 17 do gênero masculino e cinco do gênero feminino, com idades entre 5 e 10 anos (média=7,01 anos; $\mathrm{DP}=1,72$ ), submetidas à adenotonsilectomia e avaliação fonoaudiológica pré e pós-procedimento cirúrgico.

Foram incluídas crianças com hipertrofia de tonsilas palatina e faríngea, com indicação cirúrgica de adenotonsilectomia, selecionadas pelo serviço de Otorrinolaringologia Pediátrica da UNIFESP, sem realização prévia de terapia fonoaudiológica e dentro da faixa etária determinada. Foram excluídas crianças diagnosticadas com malformação craniofacial, síndromes neurológicas ou que apresentaram dificuldades em realizar as tarefas solicitadas durante as provas de avaliação.

A avaliação fonoaudiológica foi realizada por uma mesma fonoaudióloga, antes e um a seis meses após a cirurgia de adenotonsilectomia. Foi contemplado o exame das estruturas fonoarticulatórias do sistema estomatognático — lábios, língua e palato mole, e das funções - respiração (modo respiratório), deglutição (líquido), fala e fonação (qualidade vocal e ressonância). Essas estruturas e funções foram selecionadas porque, de acordo com a literatura, são as mais impactadas após a adenotonsilectomia, e devido à comum ocorrência de flacidez e alteração de posição habitual das estruturas orofaciais devido ao seu uso ${ }^{(8,9)}$.

A avaliação dos lábios foi realizada durante o repouso por meio da observação do perfil. Foram classificados em: ocluídos, quando estavam fechados em posição de repouso, sem a necessidade de contração muscular excessiva; entreabertos, quando estavam separados, sem contato entre o superior e o inferior; e abertos, quando os lábios estavam separados e com a mandíbula rebaixada ${ }^{(10)}$ ou quando foi observado a necessidade de esforço muscular necessário para o vedamento labial, tensão do músculo mentual e da musculatura labial ou perioral.

A posição de repouso da língua foi avaliada no decorrer da avaliação da fala e quando o sujeito estava em silêncio; seus lábios foram afastados pela avaliadora. Foi classificada em: normal quando permaneceu no interior dos arcos dentários durante a inspeção; ou alterada, quando permaneceu interposta entre os lábios na observação direta e/ou projetada ou interposta entre os dentes superiores e inferiores durante a inspeção da sua postura na cavidade oral.

Para avaliar o tônus labial foi solicitada a realização de movimentos de estiramento e retração labial, concomitantemente à colocação de força de contrarresistência oposta, aplicada pela pesquisadora por meio de uma espátula. O tônus foi classificado como: normal, quando apresentou resistência normal, por julgamento da avaliadora; ou alterado, quando apresentou resistência diminuída ou aumentada. Os lábios superior e inferior foram avaliados segundo os mesmos critérios. Para avaliar o tônus de língua foi solicitado às crianças que permanecessem com a boca aberta e projetassem a língua além dos lábios, sem tocar nestes ou nos dentes, momento em que foi exercida a força de contrarresistência com a espátula, a qual foi mantida firmemente pela pesquisadora ${ }^{(11)}$. O tônus da língua foi considerado: normal, quando apresentou resistência adequada ao movimento; ou alterado, quando apresentou resistência diminuída ou aumentada. $\mathrm{O}$ palato mole foi avaliado quanto à mobilidade durante a emissão das vogais /a/ e /ã/. Foi classificado como: normal, quando foi verificada uma ampla mobilidade; ou alterado, quando verificado um discreto movimento.

Quanto às funções, a deglutição de líquido foi avaliada por meio da observação direta da cavidade oral e face, com o objetivo de detectar a presença ou não de movimentos compensatórios no ato da deglutição. Foi ofertado um copo com $10 \mathrm{ml}$ de água ${ }^{(12)}$ e as crianças foram orientadas a colocar todo o líquido na cavidade oral e realizar uma deglutição de maneira habitual. Foi verificada, visualmente, a maneira como a deglutição habitual foi realizada. Classificou-se como alterada quando houve a presença de pelo menos um dos movimentos compensatórios, nomeados a seguir: interposição de língua entre as arcadas, pressionamento labial, interposição de lábios durante o ato da deglutição do líquido. A deglutição foi considerada normal quando houve ausência de contração excessiva da musculatura perioral, vedamento labial perfeito, confinamento da língua no interior dos arcos dentais e eficiência na deglutição de líquido ${ }^{(13)}$.

O modo respiratório foi classificado com: nasal quando havia uso predominante da cavidade nasal e algum ponto de vedamento da cavidade oral; oronasal quando era realizada 
ora pela cavidade nasal, ora pela cavidade oral; e oral, quando havia uso predominante da cavidade oral, complementando-se com a incapacidade da criança em manter o gole de água na boca por três minutos ${ }^{(13)}$.

A avaliação da fala foi realizada por meio da nomeação de 86 figuras, as quais continham todos os fonemas da língua portuguesa brasileira em suas variadas posições. No momento da produção, foi realizada a identificação das alterações, a partir da análise perceptivo-auditiva da pesquisadora e da inspeção visual do movimento da língua. As nomeações das figuras foram transcritas foneticamente para posterior análise. Para o presente estudo, considerou-se como alteração de fala apenas o processo de distorção ${ }^{(14)}$, por ser este um desvio fonético que pode sofrer maior influência do sistema estomatognático. Assim, a fala foi classificada em: normal, quando realizada sem qualquer distúrbio da articulação; ou alterada, quando houve a produção dos fonemas /s/ e /z/ com projeção lingual na região anterior ou lateral da arcada dentária, associada ou não à interposição lingual dos fonemas /t/, /d/, /n/ e /l/.

$\mathrm{Na}$ avaliação vocal o número de crianças participantes foi alterado, pois nessa etapa optou-se por avaliar apenas as crianças que realizaram a avaliação fonoaudiológica pós-operatória entre um mês e dois meses incompletos, a fim de evitar que um tempo prolongado para a realização da avaliação póscirúrgica comprometesse o resultado obtido. Assim, foram incluídas 15 crianças.

As crianças foram orientadas a emitir a vogal sustentada /a/ para avaliação do grau geral de desvio da qualidade vocal. Sempre que necessário, a avaliadora fazia um movimento com as mãos para que a criança mantivesse a emissão até cessar o movimento. Para a avaliação da ressonância foram orientadas a emitir uma sequência automática - contagem de números $(1-10)$. Sempre que necessário, a avaliadora emitia em intensidade baixa o número que a criança deveria dizer.

As gravações foram realizadas em uma sala silenciosa, por uma profissional especialista na área de voz que utilizou um computador da marca ITAUTEC ${ }^{\circledR}$ N8310 para gravação e armazenamento da amostra vocal e microfone profissional com condenser da marca SAMSON CSU ${ }^{\circledR}$, posicionado entre 5 e 8 centímetros da boca da criança. Todas as gravações foram registradas no Programa Sound Forge.

Três fonoaudiólogas especialistas em voz, com mais de três anos de atuação na clínica vocal, participaram como avaliadoras, em julgamento independente por meio da análise perceptivo-auditiva. Foram analisadas 33 vozes. Destas, $10 \%$ foram repetidas aleatoriamente, para confiabilidade intra-avaliadores.

As amostras vocais foram compiladas de forma aleatória. Cada criança reuniu duas gravações, pré e pós-adenotonsilectomia, compostas pela vogal sustentada /a/ para avaliação do grau geral de alteração da avaliação da qualidade vocal e sequência automática para avaliação da ressonância. As amostras foram editadas e o início da emissão foi desprezado devido à presença de instabilidade típica do ataque vocal. Por fim, o material foi gravado em CD e entregue para cada juíza responsável pela análise perceptivo-auditiva.

$\mathrm{Na}$ análise da qualidade vocal as avaliadoras foram orientadas a analisar, apenas em relação ao grau geral, se as vozes apresentaram ou não alteração, não necessitando classificá-las quanto ao tipo. Para tanto, utilizaram a escala analógico-visual $(\mathrm{EAV})^{(15)}$. Os valores de 0 a $35,5 \mathrm{~mm}$ representam a normalidade, os valores entre 35,6 a $50,5 \mathrm{~mm}$ representam um desvio vocal de grau leve, de 50,6 a 90,5 $\mathrm{mm}$ correspondem a um desvio de grau moderado, e acima de 90,6 $\mathrm{mm}$, desvio intenso. Os valores foram correlacionados da seguinte maneira: o grau 1 correspondeu à variabilidade normal da qualidade vocal (valores até $35,5 \mathrm{~mm}$ na EAV); o grau 2 representou uma estreita região para os desvios leves $(35,6$ a $50,5 \mathrm{~mm})$; o grau 3 correspondeu a uma ampla faixa para desvios moderados $(50,6$ a $90,5 \mathrm{~mm}$ ); e o grau 4 representou uma pequena faixa para os desvios intensos (acima de 90,6 mm).

Para análise das características de ressonância, as avaliadoras foram orientadas a identificar auditivamente se havia diferença entre as emissões apresentadas. Em caso de identificação de diferença de ressonância entre as emissões, as avaliadoras foram orientadas a descrever o(s) aspecto(s) mais relevante percebido(s). As emissões foram apresentadas em dupla, isto é, cada criança tinha a emissão pré e pós, as quais foram editadas e apresentadas aos juízes de forma aleatória.

Com intuito de facilitar a análise, as características ressonantais foram avaliadas quanto aos aspectos positivos, quando as juízas apontaram melhora da ressonância, classificando-as como projetada, equilibrada ou com redução da hiponasalidade ou hipernasalidade; e aspectos negativos, quando houve piora da impressão ressonantal, tanto devido à redução quanto ao aumento de nasalidade. As avaliadoras puderam ouvir as emissões quantas vezes desejassem e, posteriormente, marcaram no desenho da linha de 100 milímetros o grau da alteração vocal e em outra folha a impressão ressonantal que a emissão evidenciou.

Os resultados foram tabulados e submetidos à análise estatística, comparando-se os dados obtidos pré e pós-adenotonsilectomia. $\mathrm{O}$ teste de Wilcoxon foi utilizado para a análise relacionada às estruturas e funções do sistema estomatognático. $\mathrm{O}$ teste de McNemar foi utilizado para analisar o modo respiratório. Na avaliação da fala, foi utilizada a estatística descritiva, média, desvio padrão e a distribuição de frequência.

$\mathrm{Na}$ avaliação vocal, a estatística utilizada para medir o grau de confiabilidade entre duas variáveis foi o teste de Cronbach, o qual evidenciou valores estatisticamente elevados $(\mathrm{p}<0,005)$, permitindo inferir, que os dados apresentam consistência interna, ou seja, provido de uma amostra não viesada $(\mathrm{p}<0,001)$. O nível de significância adotado para toda análise estatística foi de $5 \%(\mathrm{p}<0,05)$.

\section{RESULTADOS}

Os dados foram agrupados em: modo respiratório; avaliação das estruturas do sistema estomatognático; função de deglutição; avaliação de fala e voz.

$\mathrm{Na}$ análise do modo respiratório houve diferença entre os dois momentos de avaliação ( $\mathrm{p}<0,001)$, com predomínio do modo respiratório nasal no pós-operatório (Tabela 1).

$\mathrm{Na}$ avaliação das estruturas e funções do sistema estomatognático, a comparação da postura de lábios apontou diferença 
Tabela 1. Comparação do modo respiratório pré e pós-adenotonsilectomia

\begin{tabular}{lccccccc}
\hline Modo & \multicolumn{2}{c}{ Oral } & \multicolumn{2}{c}{ Nasal } & \multicolumn{2}{c}{ Oronasal } & Valor de $\mathrm{p}$ \\
\cline { 2 - 8 } respiratório & $\mathrm{n}$ & $\%$ & $\mathrm{n}$ & $\%$ & $\mathrm{n}$ & $\%$ & \\
\hline Pré & 15 & 68 & 1 & 5 & 6 & 27 & \multirow{2}{*}{$0,001^{*}$} \\
Pós & 2 & 9 & 15 & 68 & 5 & 23 & \\
\hline "Valores significativos & $(\mathrm{p}<0,05)$ & - Teste McNemar & & &
\end{tabular}

$(\mathrm{p}<0,000)$ entre os momentos pré e pós-adenotonsilectomia, com maior ocorrência de postura de lábios entreabertos no pré e de lábios ocluídos no pós-operatório (Tabela 2).

Tabela 2. Comparação da postura de lábios em repouso pré e pósadenotonsilectomia

\begin{tabular}{|c|c|c|c|c|c|c|c|}
\hline \multirow{2}{*}{$\begin{array}{l}\text { Postura de } \\
\text { lábios }\end{array}$} & \multicolumn{2}{|c|}{ Ocluídos } & \multicolumn{2}{|c|}{ Entreabertos } & \multicolumn{2}{|c|}{ Abertos } & \multirow{2}{*}{ Valor de $p$} \\
\hline & $\mathrm{n}$ & $\%$ & $\mathrm{n}$ & $\%$ & $n$ & $\%$ & \\
\hline Pré & 2 & 9 & 14 & 64 & 6 & 27 & \multirow{2}{*}{$<0,000^{*}$} \\
\hline Pós & 14 & 64 & 3 & 18 & 5 & 22 & \\
\hline
\end{tabular}

${ }^{*}$ Valores significativos $(p<0,05)-$ Teste Wilcoxon

A avaliação fonoaudiológica da postura de língua em repouso apontou melhora, entre os momentos pré (predomínio de postura alterada) e pós-adenotonsilectomia (predomínio de postura de língua adequada) (Tabela 3). A diferença encontrada na análise do tônus de língua mostra que o tônus reduzido prevaleceu nos dois momentos (Tabela 3).

O tônus do lábio superior mostrou-se alterado na maioria dos casos avaliados antes da realização da adenotonsilectomia, e normalizado em 55\% dos casos avaliados após a cirurgia $(\mathrm{p}=0,637)$. $\mathrm{O}$ tônus do lábio inferior foi avaliado como alterado (reduzido) em todas as crianças antes da adenotonsilectomia e normalizado na maioria delas após o procedimento cirúrgico $(\mathrm{p}=0,479)$ (Tabela 3$)$.

A análise da mobilidade de palato mole apresentou diferença entre a avaliação pré e pós-adenotonsilectomia $(\mathrm{p}<0,000)$ (Tabela 3). Na avaliação da deglutição pré e pós-adenotonsilectomia houve diferença nos seguintes aspectos avaliados: postura de língua, mecanismo compensatório de interposição de lábios (Tabela 3).
A função de deglutição apresentou alteração em toda amostra antes do procedimento cirúrgico e se manteve alterada na maior parte da amostra após a adenotonsilectomia $(\mathrm{p}=0,108)$. $\mathrm{O}$ mecanismo compensatório de pressionamento labial mostrou-se alterado na parte da amostra nas avaliações pré e pós-adenotonsilectomia ( $\mathrm{p}=0,361)$ (Tabela 3 ).

$\mathrm{Na}$ análise da avaliação da fala foi observada a redução da ocorrência do processo de distorção na avaliação pós operatória (Tabela 4).

$\mathrm{Na}$ avaliação do grau de alteração da qualidade vocal foi verificado o predomínio do grau 1 tanto no momento pré quanto no pós-adenotonsilectomia $(\mathrm{p}=0,48)$. Ressalta-se que o grau 1 está dentro da variação da normalidade (Tabela5).

A avaliação da ressonância apontou diferença $(p<0,001)$ entre as emissões nos momentos pré e pós adenotonsilectomia. No pré, houve predomínio de aspectos negativos e, no pós, de aspectos positivos (Tabela 5).

\section{DISCUSSÃO}

A etiologia das obstruções das vias aéreas superiores é multifatorial, e quando a causa dessa obstrução é orgânica, frequentemente está relacionada à hipertrofia de tonsilas(7). Na presença de agentes que dificultam a passagem de ar pelas vias aéreas superiores, é comum haver a respiração oral de suplência ${ }^{(2,7,16)}$. As estruturas fonoarticulatórias do sistema estomatognático (lábios, língua e palato mole) e algumas funções (respiração, deglutição, fala e fonação) podem ser suscetíveis a alterações em indivíduos com diagnóstico de hipertrofia de tonsilas palatina e faríngea.

$\mathrm{Na}$ avaliação do modo respiratório pré-adenotonsilectomia, os achados evidenciaram alta ocorrência do modo respiratório oral, com melhora do padrão no momento pós-adenotonsilectomia, com maior incidência do modo nasal. A mudança do modo respiratório pode ser entendida como uma possível consequência da hipertrofia de tonsilas, com predomínio da respiração oral ${ }^{(4,7)}$. Após a intervenção cirúrgica, é possível observar mudanças do modo respiratório antes adotado, passando, na maioria dos casos, para o modo nasal, o qual é esperado fisiologicamente ${ }^{(2,17)}$.

Tabela 3. Comparação da avaliação das estruturas do sistema estomatognático e da função de deglutição pré e pós-adenotonsilectomia

\begin{tabular}{|c|c|c|c|c|c|c|c|c|c|}
\hline \multirow{3}{*}{$\begin{array}{l}\text { Estruturas do sistema } \\
\text { estomatognático e funções de } \\
\text { deglutição }\end{array}$} & \multicolumn{4}{|c|}{ Pré } & \multicolumn{4}{|c|}{ Pós } & \multirow{3}{*}{ Valor de $p$} \\
\hline & \multicolumn{2}{|c|}{ Normal } & \multicolumn{2}{|c|}{ Alterado } & \multicolumn{2}{|c|}{ Normal } & \multicolumn{2}{|c|}{ Alterado } & \\
\hline & $\mathrm{n}$ & $\%$ & $\mathrm{n}$ & $\%$ & $\mathrm{n}$ & $\%$ & $\mathrm{n}$ & $\%$ & \\
\hline Tônus da língua & 0 & 0 & 22 & 100 & 6 & 28 & 16 & 72 & $0,000^{*}$ \\
\hline Tônus do lábio superior & 7 & 32 & 15 & 68 & 13 & 59 & 9 & 41 & 0,637 \\
\hline Postura da língua na deglutição & 6 & 23 & 16 & 73 & 11 & 50 & 11 & 50 & $0,043^{*}$ \\
\hline Padrão de deglutição & 0 & 0 & 22 & 100 & 3 & 23 & 19 & 87 & 0,108 \\
\hline Interposição de lábios & 5 & 23 & 17 & 77 & 12 & 55 & 10 & 45 & $0,038^{*}$ \\
\hline Pressionamento labial & 2 & 10 & 20 & 91 & 4 & 18 & 18 & 82 & 0,361 \\
\hline
\end{tabular}


Tabela 4. Comparação pré e pós-adenotonsilectomia quanto à ocor-

rência de distorções na fala

\begin{tabular}{|c|c|c|}
\hline \multirow{2}{*}{ Fala } & \multicolumn{2}{|c|}{ Distorção } \\
\hline & média & DP \\
\hline Pré & 1,9 & 2,02 \\
\hline Pós & 1,31 & 1,64 \\
\hline Valor de $p$ & \multicolumn{2}{|c|}{0,10} \\
\hline
\end{tabular}

Tabela 5. Comparação pré e pós-adenotonsilectomia quanto ao grau geral de alteração da qualidade vocal e à impressão ressonantal evidenciada na emissão

\begin{tabular}{cccccc}
\hline \multirow{2}{*}{ EAV } & \multicolumn{2}{c}{ Pré } & \multicolumn{2}{c}{ Pós } & \multirow{2}{*}{ Valor de $\mathrm{p}$} \\
\cline { 2 - 5 } & $\mathrm{n}$ & $\%$ & $\mathrm{n}$ & $\%$ & \\
\hline Grau & 11 & 65 & 12 & 71 & \\
1 & 3 & 17 & 2 & 12 & 0,48 \\
2 & 1 & 6 & 3 & 17 & \\
3 & 2 & 12 & 0 & 0 & \\
4 & & & & & \\
Ressonância & 6 & 35 & 11 & 65 & $<0,001^{*}$ \\
Aspectos positivos & 11 & 65 & 6 & 35 & \\
Aspectos negativos & 11 & &
\end{tabular}

${ }^{*}$ Valores significativos $(p<0,05)-$ Teste McNemar

Legenda: $\mathrm{EAV}=$ escala analógico-visual

Um padrão respiratório inadequado pode proporcionar uma série de cortejos de adaptações posturais e alterações de funções do sistema estomatognático. Devido à redução do espaço da orofaringe, a língua adota uma postura anteriorizada ${ }^{(4,16,18)}$, como verificado neste estudo antes da intervenção cirúrgica. Os dados da avaliação pós-adenotonsilectomia indicaram postura de língua adequada em repouso, ou seja, dentro da cavidade oral, na maior parte da amostra. O novo posicionamento da língua é uma consequência da adequação do modo respiratório procedente do maior espaço orofaríngeo decorrente da exerese das tonsilas ${ }^{(2,6,17)}$.

Outras alterações morfológicas comumente presentes nos respiradores orais são a postura de lábios entreabertos ou abertos e a hipotonia de face, em especial de lábios e língua $^{(2,6,7)}$. Como resultado da avaliação da postura de língua em repouso verificou-se maior incidência, pré-adenotonsilectomia, de lábios entreabertos, seguida de lábios abertos. Pós-adenotonsilectomia, foi observada uma mudança postural, prevalecendo à posição de lábios ocluídos.

A manutenção de postura de lábios inadequada é justificada muitas vezes pela obstrução de vias aéreas, decorrente da hipertrofia de tonsilas ${ }^{(19-20)}$. A melhora da postura de lábios surge mediante a possibilidade da adoção de um padrão respiratório fisiologicamente mais adequado após a exerese das tonsilas, tornando possível haver uma reorganização de alguns órgãos fonoarticulatórios, principalmente no que diz respeito à postura dos articuladores, uma vez que devido a respiração nasal o vedamento labial se torna possível ${ }^{(2,17)}$.

A avaliação do tônus labial evidenciou a redução de tônus labial superior e inferior antes da adenotonsilectomia, com manutenção de tônus reduzido no lábio inferior e adequação do tônus de lábio superior após a cirurgia. Já o tônus de língua manteve-se alterado na maioria das crianças nos momentos pré e pós-adenotonsilectomia. Contudo, houve discreta melhora após a cirurgia.

É referida na literatura ${ }^{(17)}$ uma possível melhora miofuncional espontânea nos primeiros meses após a adenotonsilectomia. Hipotetizou-se que uma nova condição orofaríngea possibilite uma melhora espontânea em relação à postura, pois o fator principal de manutenção do padrão inadequado de postura de língua e de respiração foi eliminado.

A fisiologia muscular descreve que diversos estímulos atuam sobre o músculo esquelético, promovendo alterações fisiológicas e moleculares, os quais colaboraram para modificar a atividade contrátil do músculo a partir da reorganização de eventos celulares. Essas mudanças estão diretamente envolvidas na função mecânica da fibra muscular, convergindo para a melhora da contração e para geração de força, permitindo, assim, que as fibras da musculatura esquelética se adaptem ao estímulo, fenômeno denominado plasticidade muscular ${ }^{(21)}$. Esse efeito remete a hipótese de que a melhora encontrada no tônus de lábio superior e de língua pode ter ocorrido devido ao fator plasticidade muscular, pois os músculos mantêm-se normalmente em um estado de contração parcial e a ação de um estímulo externo extensivo (no caso a respiração nasal, os lábios ocluídos e a postura de língua adequada) acima do limiar desencadeia uma reação no músculo ${ }^{(22)}$.

A mobilidade de palato mole pode ficar comprometida, isto é, reduzida devido ao aumento de massa na região orofaríngea. Contudo, após a realização da adenotonsilectomia espera-se que a mobilidade seja restabelecida evidenciando melhora da fala e da $\mathrm{voz}^{(3,4,16)}$.

Considera-se como deglutição normal a ausência da utilização dos músculos da expressão facial e língua contida na cavidade oral. Qualquer mudança observada antes ou durante a deglutição indica uma alteração nesta função. Mecanismos compensatórios como pressionamento labial, ação mentual e interposição de língua podem estar presentes na deglutição classificada como alterada ${ }^{(8-9)}$. Na tentativa de corrigir estas alterações, mecanismos compensatórios de interposição labial e pressionamento labial se fazem presentes, agindo de forma mais ativa na busca do selamento labial para a adequação da respiração ${ }^{(7-9)}$.

A literatura ${ }^{(6,17)}$ refere haver normalização da deglutição no período de um a seis meses após o procedimento cirúrgico e sem intervenção fonoaudiológica. Contudo, nosso estudo mostrou que a deglutição alterada persistiu na maior parte da amostra.

Prováveis alterações nas estruturas fonoarticulatórias podem comprometer a inteligibilidade da fala tanto em termos de articulação quanto na voz, já que a participação adequada dos articuladores móveis e fixos do sistema estomatognático para execução da fala é indispensável. Por esse motivo, este estudo englobou a pesquisa da fala e de algumas características da voz pertinentes ao tema abordado (presença ou ausência de alteração da qualidade vocal e ressonância).

A presença de desvios na articulação decorrente de forma ou função alterada das estruturas estomatognáticas constitui um tipo de distúrbio articulatório( ${ }^{(23)}$. A distorção na produção dos fonemas /s/, /z/,/t/,/d/, /n/, /l/ com presença de interposição 
de língua ${ }^{(14)}$ podem estar presentes nos quadros de respiração oral $^{(4,7,24)}$, alteração dos articuladores ${ }^{(24)}$, principalmente da língua, e alteração da função de deglutição ${ }^{(4,7)}$.

Encontra-se descrita na literatura uma variação quanto à faixa etária para o processo de aquisição fonêmica. Para o presente estudo, optou-se pela faixa etária que compreende dos 3 aos 5 anos de idade ${ }^{(25)}$, pois o atual processo de alfabetização se inicia na pré-escola, quando as crianças estão com 5 anos, e, devido a esse fator, tanto a escola quanto os pais buscam por auxílio fonoaudiológico mediante quadros de imprecisão articulatória. Por esse motivo o presente estudo foi iniciado nessa faixa etária e adotou 5 anos como limite para aquisição fonêmica.

A síndrome do respirador oral também pode provocar um comprometimento na voz, principalmente em relação à qualidade vocal e ressonância, uma vez que faringe, língua, palato, cavidade oral e nariz agem como cavidades de ressonância e são, em parte, responsáveis pela qualidade vocal ${ }^{(26)}$. A análise global da qualidade vocal, baseada na EAV, apontou que a voz maior parte das crianças foi classificada como grau 1, ou seja, dentro do padrão de normalidade, tanto no momento pré quanto no pós- adenotonsilectomia.

Buscando verificar a impressão que a ressonância pode causar nos momentos pré e pós-adenotonsilectomia, as impressões analisadas pelas juízas foram nomeadas quanto aos aspectos positivos (emissão clara e equilibrada) e negativos (qualquer evento que interferisse na projeção da emissão). Os resultados das análises mostraram maior incidência de aspectos negativos no momento pré-adenotonsilectomia, e maior ocorrência de aspectos positivos no momento póscirúrgico. $\mathrm{O}$ aspecto negativo mais referenciado pelas juízas foi a presença de hiponasalidade, seguido de hipernasalidade, em menor proporção. $\mathrm{O}$ aspecto positivo mais citado pelas juízas foi a emissão projetada e equilibrada.

Há relatos de casos que citam a presença de hipernasalidade após a intervenção cirúrgica ${ }^{(2)}$, dado esse não evidenciado nessa amostra. A melhora das características ressonantais pode ser justificada pelo aumento da amplitude de energia em decorrência da redução da área de superfície do tecido e do aumento da passagem nasal após a cirurgia ${ }^{(27)}$, reduzindo o amortecimento acústico $^{(28)}$, associados a adequada mobilidade de palato ${ }^{(4,5,16)}$.

O estudo sobre os aspectos fonoaudiológicos pré e pós-adenotonsilectomia permite inferir que a hipertrofia de tonsilas palatina e faríngea causa alterações, de graus e impactos variados, na tonicidade e postura de lábios e língua e na mobilidade do palato mole, interferindo na execução das funções de deglutição, fala e fonação.

Quando o fator causal da obstrução das vias aéreas superiores é suprimido, é possível observar, em alguns casos, melhora espontânea em alguns dos aspectos. Contudo, neste estudo, essa melhora não foi suficiente para adequar as condições de todos os aspectos avaliados, e foi necessário encaminhar 19 das 22 crianças para atendimento fonoaudiológico.

Faz-se, ainda, nesta finalização, uma ressalva sobre a avaliação clínica das estruturas e funções do sistema estomatognático, a qual é comumente realizada de forma subjetiva e pode haver variações conforme experiência clínica do avaliador. A presença de um grupo controle talvez fosse importante para ajudar a reduzir o viés da subjetividade presente neste tipo de avaliação.

Assim, faz-se necessária a avaliação fonoaudiológica após a intervenção cirúrgica, para reavaliação das estruturas e funções do sistema estomatognático. No pós-operatório, em caso de manutenção dos aspectos alterados, deve-se encaminhar a criança para atendimento fonoaudiológico, visando à correção de tais inadequações.

\section{CONCLUSÃO}

Frente aos achados desse estudo pode-se concluir que após a adenotonsilectomia algumas estruturas e funções podem se readaptar ou apresentar melhora espontaneamente. Contudo, verifica-se que para muitas crianças é necessário o encaminhamento para o atendimento fonoaudiológico para a readaptação das estruturas estomatognáticas e funções avaliadas. Dessa forma, faz-se necessário considerar uma avaliação fonoaudiológica após a adenotonsilectomia para analisar as condições das estruturas e funções do sistema estomatognático.

* FBAB foi responsável pela coleta, tabulação e análise dos dados, revisão científica e redação do manuscrito; $R A$ foi responsável pela análise dos dados, verificação do método, da estatística, revisão científica e revisão final; BMC foi responsável pela revisão científica, verificação do método, revisão final e revisão ortográfica.

\section{REFERÊNCIAS}

1. Carvalhal ML, Castagno LA. Hipertrofia da amígdala faríngea: clínica e cirúrgica. Rev Bras Otorrinolaringol. 1986;52(2):16-9.

2. Junqueira P, Vaz ACN, López CP, Pignatari SN, Weckx LLM. Técnica de correção de hipernasalidade causada por adenoidectomia. Ver Bras Otorrinolaringol. 2002;68(4):593-6.

3. Salami A, Jankowska B, Dellepiane M, Crippa B, Mora R. The impact of tonsillectomy with or without adenoidectomy on speech and voice. Int J Pediatr Otorhinolaryngol. 2008;72(9):1377-84.

4. Mora R, Jankowaska B, Mora F, Crippa B, Dellepine M, Salami A. Effects of tonsillectomy on speech and voice. J Voice. 2009S ep;23(5):614-8.

5. Bertino G, Matti E, Migliazzi S, Pagella F, Tinelli C, Benazzo M. Acoustic changes in voice after surgery for snoring: preliminary results. Acta Otorhinolaryngol Ital. 2006;26(2):110-4.

6. Lundeborg I, McAllister A, Graf J, Ericsson E, Hultcrantz E. Oral motor dysfunction in children with adenotonsillar hypertrophy-effects of surgery. Logoped Phoniatr Vocol. 2009;34(3):111-6.

7. Valera FC, Travitzki LV, Mattar SE, Matsumoto MA, Elias AM, Anselmo-lima WT. Muscular, functional and orthodontic changes in pre school children with enlarged adenoids and tonsils. Int J Pediatr Otorhinolaryngol. 2003;67(7):761-70.

8. Bianchini AP, Guedes ZCF, Vieira MM. Estudo da relação entre a respiração oral e o tipo facial. Rev Bras Otorrinolaringol. 2007; 73(4):500-5.

9. Lemos CM, Wilhelmsen NSW, Mion OG, Mello Júnior JF. Alterações funcionais do sistema estomatognático em pacientes com rinite alérgica. Rev Bras Otorrinolaringol. 2009;75(2):268-74.

10. Cattoni DM, Fernandes FDM, Di Francesco RC, Latorre MRDO. Características do sistema estomatognático de crianças respiradoras orais: enfoque antroposcópico. Pro Fono. 2007;19(4):347-51.

11. Daniels L, Worthigham C. Provas de função muscular: técnicas de exame manual. 6a ed. Rio de Janeiro: Guanabara Koogan; 1996. 
12. Falda V, Guimarães A, Bérzin F. Eletromiografia dos músculos masseteres e temporais durante deglutição e mastigação. Rev Assoc Paul Cir Dent. 1998;52(2):151-7.

13. Junqueira P. Avaliação miofuncional. In: Marchesan IQ. Fundamentos em fonoaudiologia: aspectos clínicos da motricidade oral. Rio de Janeiro: Guanabara- Koogan; 1998. p. 13-21.

14. Yavas MS, Hernandorena CLM, Lamprecht RR. Avaliação fonológica da criança: reeducação e terapia. Porto Alegre: Artes Médicas; 1992.

15. Yamasaki R, Leão S, Madazio G, Padovani M, Azevedo R, Behlau M. Correspondência entre escala analógico-visual e a escala numérica na avaliação perceptivo-auditiva de vozes. In: $16^{\circ}$ Congresso Brasileiro de Fonoaudiologia; 2008. Set 24-27. Campos do Jordão. Anais Sociedade Brasileira de Fonoaudiologia; Campos do Jordão, São Paulo; 2008. p. 1080

16. Mora R, Jankowaska B, Mora F, Crippa B, Dellepine M, Salami A. Effects of adenotonsillectomy on speech spectrum in children. Int $\mathrm{J}$ Pediatric Otorhinolaryngol. 2007;71(8):1299-304.

17. Valera FC, Travitzki LV, Anselmo-lima WT. Myofunctional evaluation after surgery for tonsils hypertrophy and its correlation to breathing pattern: a 2-year-follow up. Int J Pediatric Otorhinolaryngol. 2006;70(2):221-5.

18. Kawashima S, Peltomaki T, Sakata H, Mori K, Happonen RP, Ronning O. Craniofacial morphology in preschool children with sleeprelated breathing disorder and hypertrophy of tonsils. Acta Paediatr. 2002;91(1):71-7.

19.Menezes VA, Leal RB, Pessoa RS, Pontes RMES. Prevalência e fatores associados à respiração oral em escolares participantes do projeto Santo Amaro - Recife, 2005. Rev Bras Otorrinolaringol. 2006;72(3):394-9.
20. Schimit R. Caracterização da postura, tônus e mobilidade de lábios e língua em crianças respiradoras orais. [Dissertação] São Paulo: Universidade Católica de São Paulo - PUC/SP;2010: 59p.

21. Boff SR. A fibra muscular e fatores que interferem no seu fenótipo. Acta Fisiátrica. 2008;15(2):111-6.

22. Lopes CL. Qualidade Vocal Consultoria: tipos de ação muscular (cap. III) Paraná; 2008. [Internet]. [atualizado 2008 Abr 18]. Disponível em: http://qualidadevocal-fonoaudiologo.blogspot.com.br/2008/04/tipos-deao-muscular-cap-iii.html

23. Tomé MC, Farias SR, Araújo SM, Schimit BE. Ceceio interdental e alterações oclusais em crianças de 03 a 06 anos. Pro Fono. 2004;16(1):19-30.

24. Farias SR, Ávila CRB, Vieira MM. Relação entre fala, tônus e praxia não verbal do sistema estomatognático em pré-escolares. Pro Fono. 2006;18(3):267-76

25. Andrade CRF. Prevalência das desordens idiopáticas da fala e da linguagem em crianças de um a onze anos de idade. Rev Saúde Pública. 1997;31(5):495-501.

26. Sataloff TS. Anatomia funcional e fisiologia da voz. In: Sataloff TS, Gould WJ, Spiegel JR. Manual prático de oncocirurgia. Rio de Janeiro: Revinter; 2002. p. 63-176.

27. Chuma AV, Cacae AT, Rosen R, Feustel P, Koltaii PJ. Effects of tonsillectomy and/ or adenoidectomy on vocal function: laryngeal, supralaryngeal and perceptual characteristics. Int J Pediatr Otorhinolaryngol. 1999;(47):1-9.

28. Behrman A, Shikowitz MJ, Dailey S. The effect of upper airway surgery on voice. Otolaryngol Head Neck Surg. 2002;127(1):36-42. 